\title{
Acoustic Information During Motor Control and Action Perception: A Review
}

\author{
Alexandra Pizzera ${ }^{1,3, *}$ and Tanja Hohmann ${ }^{1,2}$ \\ ${ }^{1}$ Institute of Psychology, German Sport University Cologne, Köln, Germany; ${ }^{2}$ Institute of Sport Science, University of \\ Stuttgart, Stuttgart, Germany; ${ }^{3}$ Institute of Sports and Sports Sciences, Heidelberg University, Heidelberg, Germany
}

\begin{abstract}
There is a whole body of research that provides evidence that the motor system plays a crucial role in controlling as well as perceiving movements. So far a lot of evidence for the interaction of action and perception derives from studies in the visual domain. However, up to now not much is known about the role of acoustic information. The focus of this review is to provide an overview regarding the role of the motor system and auditory sense during action perception and motor control. Recent theories and studies that discuss the interaction of perception and action will be reviewed with an emphasis on the use of acoustic information. Empirical evidence derived from behavioral as well as neuroscience research using simple as well as whole-body movements will be provided. Additionally, we will provide perspectives regarding future research questions to bring forward our understanding of the role of acoustic information in the control and perception of actions and its underlying mechanisms.
\end{abstract}

Keywords: Action control, auditory information, authorship, visual perception.

\section{INTRODUCTION}

Imagine a hurdler who is preparing for a competition. As he is running on a track and jumping over the hurdles he hears the footsteps resulting from the foot-ground interaction. At the same time, he might feel the characteristics of the ground, the forces resulting from the pressure he puts on the ground or the different joint angles he might have adapted according to the demands of the environment. In addition, he might also see somebody else performing these actions and hear a coach who gives acoustic feedback in form of a rhythmical clapping of the hands regarding the correct step sequence. Afterwards the coach may present a video to the athlete to give him feedback regarding his performance.

This example nicely illustrates the different dimensions when thinking about the role of auditory cues for motor control as well as for action perception. The first dimension refers to the authorship of an auditory cue. The hurdler perceives the sound of his own steps during running (perception of own movements). If he watches another athlete performing the task, he perceives the sound of another person's footsteps (perception of other individual's movements). The second dimension applies to the timing of acoustic perception. While our sprinter is performing the task, he perceives his stepping sounds at the same time as he is performing the task (online perception). If he is looking at his performance in the video, he perceives the sounds of his steps after the real execution (offline perception). The third dimension deals with the kind of feedback that is provided by the auditory cues. If the hurdler perceives the sounds of his steps while running, he gets feedback from his body about the movement (internal feedback). One then speaks of natural movement sounds.

\footnotetext{
*Address correspondence to this author at the Institute of Psychology, German Sport University Cologne, Köln, Germany; Tel: +49-221-4982-5721; Fax: +49-221-4982-8320; E-mail: A.Pizzera@dshs-koeln.de
}

If the coach claps his hands to provide information regarding the correct rhythm of the step sequence, our athlete receives auditory feedback from another person (external feedback). This external feedback can be either based on natural movement sounds from the coach or on artificial sounds, using for instance a tambourine to imitate the correct step sequence. In this context, the hurdler not only perceives the sound of his steps, he might also use this cue for movement control. He might want to imitate the sound provided by the coach's hand clapping with his feet. So he might use this cue for motor control and learning as well.

The question on how we perceive actions in the environment as well as how we control our own movements by using multimodal cues (e.g. vision, audition, touch) to act appropriately is the aim of a vast body of research [1-3]. There are different accounts to explain the interaction of perception and action. The "sensorimotor view" postulates that actions are "reactions" to certain stimuli in the environment ("stimulus triggered hypothesis" [4]). For example if an individual hears a dragging sound of the foot on the ground, this may cause the individual to lift up the foot a little bit higher during the next footstep. The "ideomotor view" assumes that an internal mechanism like a certain goal that the individual wants to achieve, provokes an action. It is the goal of the action that dictates the planning of that action ("goal triggered hypothesis" [4]). Crucial for the control of upcoming actions are the anticipated action effects. A piano player wants to play a certain piece of music and therefore he has to move his fingers in a predetermined sequence. This presupposes that the "system" already knows what kind of movements are necessary to achieve a certain goal.

The "Theory of internal models" as well as the "Theory of event coding" (TEC) describe how actions are planned in terms of their intended goals [4]. Two functionally different systems are used to plan actions based on intended action 
effects: the "forward model" and the "inverse model". To specify which motor commands are necessary to achieve an intended effect in the environment the forward model is used. To specify which effect was caused by which actions the so called "inverse model" is used. Additionally it is assumed that predictions concerning the sensory consequences of the action are derived from the motor command if a movement is planned or even if it is executed. The assumption that the human motor system acts as an anticipatory mechanism is rooted in the "reafference principle" [5].

According to this principle the prediction of sensory consequences of an action has several functions. Predictions about sensory events produced by self-induced motion such as head movements are necessary to cancel out these sensory consequences originating from that motion to achieve a perception of consistency. Additionally, the prediction of sensory consequences is important to guide an ongoing movement.

The forward model is used to assign perceived sensory events to the own person [6]. If the modulated events as well as the perceived sensory events are similar, the actor perceives the action as an own action. Therefore, if our hurdler is running on the track, his motor system makes predictions about the upcoming movement sounds. If these sounds correspond with the actual movement sounds, he perceives these movements as his own. Additionally, TEC postulates that actions are planned in terms of their anticipated or intended effects. Therefore, our hurdler could have in mind what he should hear when running on the track.

Whereas early frameworks assumed that processes of action planning and action control are separated and independent from each other [7], recent frameworks postulate nearly no differences between perceptual and motor representations. Action as well as perception are coded in the same medium ("Common-Coding Theory") [8]. It is assumed that there is an overlap between the preparation and execution of an action, the imagination as well as the perception of an action. Action representations and perceptual representations influence each other. There is plenty of evidence for the close connection between perception and action. On the one hand neuroscientific studies were conducted with the aim to provide evidence for the interaction of perception and action on a neurophysiological level. The discovery of a mirror neuron system in monkeys as well as humans was regarded as a neurophysiological basis for the direct link between action and perception. A particular class of visuomotor neurons discharges both when the animal or the human conducts a particular action as well as when he observes another individual (monkey or human) performing a similar action [9, 10]. Furthermore it was shown that the observation or the imagination of an action excites the motor programs that are used to execute that same action $[8,11]$. During action perception there is re-activation of sensorimotor memory representations ("direct-matching hypothesis") [12]. However, it should be mentioned that there is currently a debate regarding the precise nature of the regional activity and whether similar activations in certain neural regions in the brain during the execution, imagination and perception of a movement really mean that perception and action are nearly simi$\operatorname{lar}[13]$.
On the other hand behavioral studies were conducted showing that through the involvement of the own motor system during perception, movements can be interpreted and anticipated correctly. Empirical evidence for the interaction of perception and action and the role of the motor system is supported by several investigations dealing with motor experience, motor intention, motor learning, and motor competence in the visual domain (for an overview, see [3]).

However, up to now research regarding the interaction of perception and action has focused mainly on the visual domain. There is not a lot of empirical evidence about the role of acoustic information regarding the interplay of perception and action. However, it seems to be fruitful to examine the role of acoustic information, because rhythm is a basic principle of actions and this can be optimally represented via acoustic information [14]. Furthermore, an action, whether it is a target-orientated fine motor grasping action, an aimlessly gross motor walking activity or instinctive dancing to the favorite music, generates an auditory product. Movement and sound are therewith rather directly connected; even the physical explanation for noise is a moving sound wave. The variation of sounds caused by movement is thereby inexhaustible. For instance, when individuals walk, they usually make sounds, resulting from the contact phase between the feet and the ground. Such footstep sounds can contain valuable information about the walking person (e.g. sex or mood), but also on the surface he or she is walking on [15, 16]. The aim of the review will be to summarize behavioral as well as neuroscientific findings regarding the role of auditory cues (action-related sounds) in motor control as well as in action perception. Using the three dimensions described above, research will be presented regarding the question on how auditory cues are used for the control of actions and action perception. Within the review we will focus on actionrelated natural movement sounds (for an overview of the studies presented, see Table 1). Studies that deal with speech, language, rehabilitation or computerized sounds derived through movement (button presses) will be excluded.

\section{THE ROLE OF AUDITORY CUES FOR ACTION PERCEPTION: THE AUTHORSHIP (OWN VS OTHER) AND THE TIMING OF ACOUSTIC INFOR- MATION (ONLINE VS OFFLINE)}

The authorship refers to the person who is responsible for a certain action. The question is whether a certain action was caused by the own movement or by someone else. The timing of acoustic information can be described two-fold. First, timing can refer to action and perception processes and whether these two occur simultaneously. In this context, online effects are defined as perceptual and motor processes occurring simultaneously or with a minimum delay of less than three seconds. Offline effects describe temporally separated perception and action processes [3]. Second, timing can refer to perceptual processing of action only and how the different senses interact. For instance, the question can be raised whether acoustic information is temporally in synchrony with visual motion perception or not. There are two lines of research dealing with authorship effects in action perception. On the one hand researchers examined if participants are able to recognize own past movements (offline 
Table 1. Selected experimental studies using online or offline perception paradigms, with or without natural movement sounds, and either pure auditory or combined visual and auditory information. Grey shading indicates neuroscientific studies.

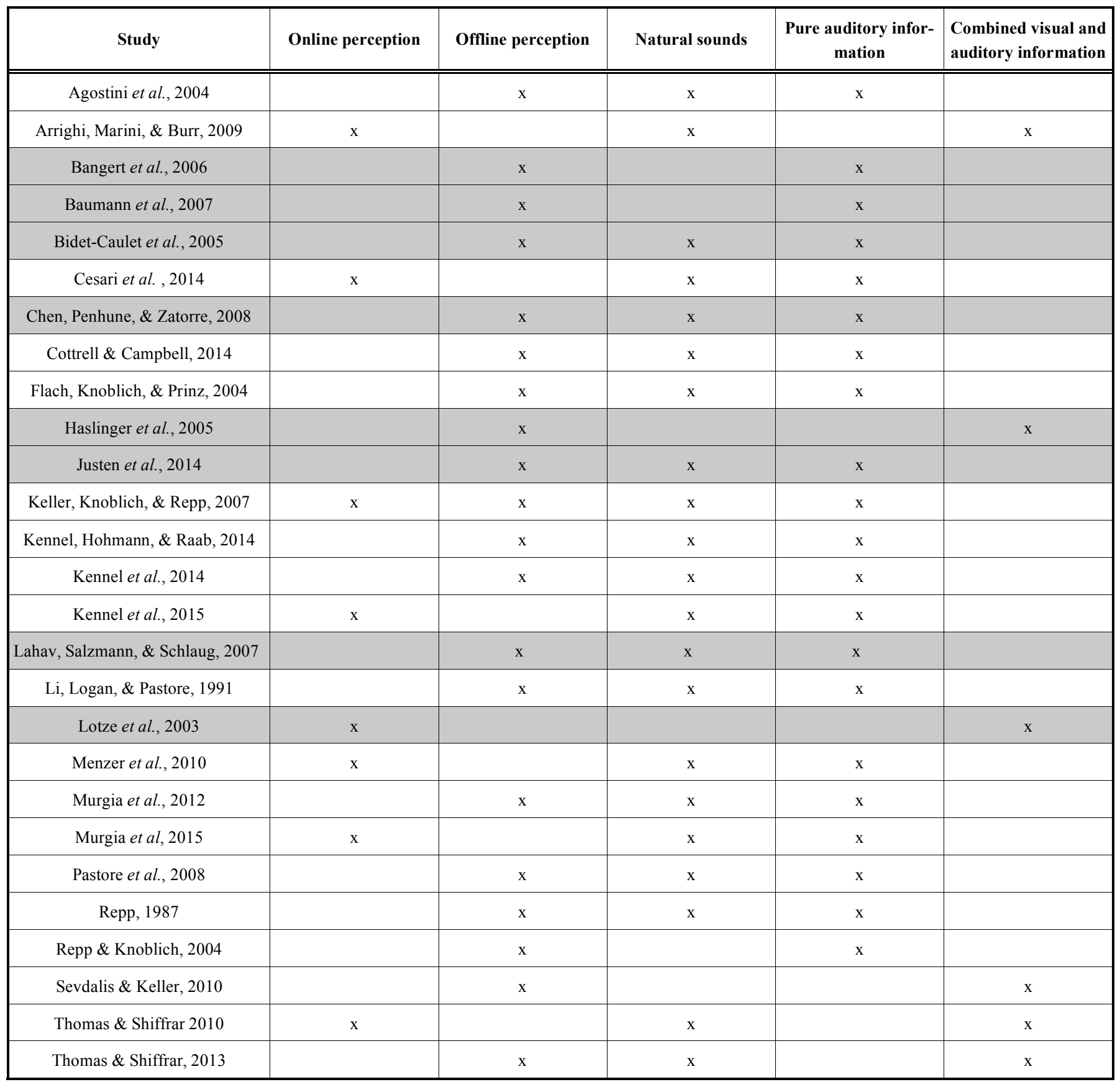

perception of own and other individuals' movements). On the other hand studies investigated at which point an individual perceives an action effect as caused by the own movement and at which point we perceive that a movement was caused by somebody else (online perception of own and other individuals' movements).

\section{Offline Perception of Own and Other Individuals' Move- ments}

\section{Perception of Other Individuals’ Movements}

Studies provide evidence that individuals are quite accurate (average of $96 \%$ ) in perceiving the motion direction of a walker moving across the auditory scene of a perceiver (left to right or vice versa) when listening to his or her footsteps [17]. In addition, studies have shown that individuals are able to identify posture [18] and gender [19] from movement sound. The gender of a walking individual was identified correctly on $82 \%$ of the trials. A further analysis of the specific sounds generated during walking revealed that the stance phase tends to be shorter in females compared to males, although walking pace is not significantly different. The authors concluded that listeners are sensitive to the temporal pattern of walking. In this context, Cottrell and Campbell [20] took up this idea by investigating the cadence of a walker, which is defined as the rhythm of a walker moving 
across a surface. It incorporates the duration of the swing and stance phase and any subtle differences between the movement of the left and right foot. These inter-foot-strike intervals and left right patterning can give an indication about the size and stride length of the walker. The results revealed that participants were able to accurately distinguish between biological and non-biological (ball bouncing, drumbeats) motion, however, using temporal as well as nontemporal factors such as the spectral pattern and energy level of the individual impact sounds. Therefore, cadence was one cue used for acoustic action perception, but not the only one.

\section{Perception of Own and Other Individuals' Movements}

The idea behind this line of research is to provide evidence that our own motor system is used for perception. A high "resonance" is assumed whenever an individual perceives own movements. In that case the same "system" that has planned the action now perceives exactly that action. It is assumed that some kind of familiarity is awaked in the observer. The overlap between perception and action is assumed to be smaller if a stranger is observed. Activity within the parietal premotor circuit starts earlier when own movements are judged in reference to other individuals' movements [21]. Most of the previous research investigated the effect using visual cues (e.g. point-light method [22]), however, auditory action consequences may differ in their effects. Specifically, temporal discrepancies may be better detectable from auditory cues and therefore are powerful agency cues. Repp [23] conducted a series of experiments with the aim to explore whether individuals are sensitive to spectral properties of claps. Within the first experiment one aim was to examine whether the listeners were able to recognize the sex and the hand size of the person who was clapping. The second aim was to explore whether participants were able to identify their own past clapping sounds. Results showed that individuals were able to identify their own past clappings, but they were not able to distinguish between male and female claps. In line with the acoustic analysis of the clap spectrum and the behavioral data, hand size did not seem to be an important cue. Within the second experiment the aim was to examine whether participants were able to distinguish between several hand configurations. Results provide evidence that participants were able to distinguish between eight different clapping styles. Flach, Knoblich, and Prinz [24] tried to replicate the findings of Repp [23]. The aim of a first study was to demonstrate that acoustic recordings of clapping provide sufficient information to be able to discriminate between the own performance and that of other individuals. The task was to decide whether a certain clapping sequence was a past own performance or that of another individual. The results of this study clearly demonstrated that individuals can recognize their own past clapping even if the claps are reproduced by uniform tones so that only the temporal pattern can be perceived. This was achieved by removing cues like acoustic differences that may evolve because individuals used different hand positions for clapping, while retaining the general tempo (speed with which participants clapped their hands) and the relative timing (rhythmical information). Interestingly, no difference between the current and previous study were found. A second study was conducted with the aim to clarify which cues individuals use to identify their own clapping [24]. The original timing remains unaffected whereas the relative timing was changed. The tones were replayed either in the participant's own tempo or in the tempo of another person. After that manipulation the participants were not able to identify their own clapping anymore. This leads to the assumption that for selfrecognition the relative timing of the tones as well as the general tempo are important cues to be able to identify own past performances. However, the main task in the reported studies was to identify own versus other movements with regard to discrete movements or sequences of discrete movements. The question arises if similar results would be found when using continuous movements. This possible connection to the nature of the task remains unanswered and might stimulate further research to untangle if action recognition through acoustic information may depend on specific kinematic components of the action.

Another possibility to get further insight into the auditory action-perception link is through the use of expert performers, who have a quite established and specialized action repertoire. This idea of a strong connection between expert actions and their resulting events was addressed in a study by Repp and Knoblich [25]. The aim of the study was to examine whether skilled piano players were able to identify own past pieces of music. 12 piano players with several years of experience took part in the study. In the first part of the study their task was to play different, unfamiliar music sequences (duration 15 to 20s) on a keyboard. On half of the trials a silent keyboard was used. The participants could not hear the music. In the second part of the study (two months later) the piano players listened to the music pieces that were recorded in the first session. The participants had to rate on a 5-point scale $(1=$ not me, $5=$ me) whether they listened to an own piece of music. Because own past products were rated higher than those of other piano players, further evidence is provided for the assumption that perception and action are closely linked. Given these results, Repp and Knoblich [25] conducted several follow up experiments to examine what cues individuals use to identify own past products. Even when the authors manipulated cues like the speed as well as the overall dynamic level of the captured music pieces or they removed dynamic nuances within the pieces of music, no decrement in performance was found. It seems that participants still have enough information to be able to recognize own past actions.

Whereas in the above described studies the production of a certain sound is the aim of the movements, recent research focused on auditory information that arises as a byproduct of the movement. For instance, it was shown that golfers were able to identify own past movements, except when the other person's sounds had nearly the same temporal factors [26]. Temporal factors were manipulated by adapting or increasing either the relative timing (relation between the duration of the upswing and downswing motion of the golfer) or the overall duration of the swing. Similar results were reported in a study with hurdlers [27]. The participants' own movement sounds during hurdling were recorded. Subsequently, they were asked to perform discrimination and identification tasks based on movement sounds from themselves and others. The participants were able to discriminate identical and different sound pairs, independent of the agent. In addition, 
they identified their own movement sounds significantly better than strangers' sounds, indicating the close link between perception and action based on auditory reafferences. In order to investigate the specific sound features that may contribute to auditory action perception, previously recorded hurdling sounds were presented to participants, however, manipulating the rhythmic step structure and amplitude range [28]. Similar to the studies reported before, participants were able to discriminate between own and other movements, independent of the manipulation. The authors suggested that sound identification may be processed holistically as an auditory gestalt. What exactly could be defined as a grouping principle is unknown so far. Researchers have discussed auditory stimuli perceived as a holistic unit that represents a stream across time for audition [29, 30]. However, if this unit represents kinematic information or different sources of temporal information or any other factors awaits further investigation.

\section{Online Perception of Own and Other Individuals' Movements}

Studies have shown that such agency judgments are influenced by manipulating perceptual and sensorimotor cues [e.g. 22, 31, 32]. Questions on how an individual might know if he or she is causing the footsteps or at which point he or she begins to suspect that the sounds are caused by someone else are subject of investigations. In this context, a study was conducted in which participants were instructed to walk at a normal and relaxed speed in a clockwise circuit [33]. During walking, they received auditory feedback on their footsteps, which were temporally delayed ranging from 16 to $1800 \mathrm{~ms}$. Using a handheld wireless device, participants were then asked to indicate if the walking that they heard over the headphones corresponded to the walking they had just performed. The results revealed that agency judgments depended on the delay. With very short delays of 16 to $100 \mathrm{~ms}$ (auditory feedback almost similar to real-time footsteps) participants achieved up to $90 \%$ confirmatory agency judgments, which decreased to 34 and $28 \%$ for delays of 250-450 ms. Percentage of agency judgments increased again for 450-750 ms delays. The same sinusoidal pattern of agency judgments was repeated for delays between 750 and 1300 ms. In line with research on pianists, the participants' agency judgments increased, as the delays approached the time of the subsequent actual footstep. This support the idea that less disruption occurs when relationships between perception and action form simple phase ratios as opposed to asynchronous feedback that disrupts produced timing (for a review and discussion on the coordination of perception and action specifically in music, see Pfordresher [34]). The authors of the walking study further concluded that mechanisms of delay-related agency are similar whether auditory or visual modalities are tested. In addition, using gait analysis, the authors suggested that conscious gait monitoring also depends on the participant's gait period, however, independent of pedal crossover.

With regard to synchrony in perceptual processing, a study using taps in tap dancing as sounds demonstrated that movement sounds need to be in synchrony (temporally coincident) with the visual display, showing the functional combination of audiovisual information [35]. Participants were asked to detect tap dance sequences under conditions involving no sound, desynchronized audio and visual displays (videos shown as point-light displays) or synchronized displays. The results confirmed that synchrony between auditory and visual stimuli can improve visual performance. However, a study with point-light displays showing dancing, walking and clapping movements without music, in synchrony or out of synchrony with music contrasted these results [36]. Although self recognition was better than chance in all conditions, accuracy did not increase with auditory information. This suggests that agent identification seems to depend much more on motor cues (visual information about personal movement kinematics) than on auditory or audiovisual information. Similarly, a study in which participants heard synchronous or out-of-phase-footsteps with point-light displays of walking individuals revealed no benefit in visual sensitivity when hearing synchronous footsteps [37, experiment 1]. Due to this inconsistency with the study by Arrighi, Marini and Burr [35], the authors followed up on this idea by taking into account the meaningfulness of sounds, which will be presented in the section on the feedback type of acoustic information.

Online effects in motor control were shown in a study using skateboarding as a task. Participants were instructed to anticipate and simulate a skateboarding jump by listening to the sound it produces [38]. Measuring underfoot forces and postural adjustment, the authors revealed that only skaters were able to use the auditory information to guide proper anticipations of the corresponding movement patterns. Other effects of real-time auditory information on motor control were shown in a study by Menzer et al. [33]. Delayed auditory feedback systematically changed the walking speed and gait period of individuals. With respect to our introductory example, a very recent study revealed that even during complex whole-body movements such as hurdling, auditory feedback influences the participants' movements [39]. Using an online paradigm, participants either heard their normal step sounds during running, white noise in which sound was masked or a delayed auditory feedback. Using a selfinvented feedback apparatus worn as a belt around the waist, the participants' step sounds were recorded and immediately given as auditory feedback to the participants while running, however, with a $180 \mathrm{~ms}$ delay. This delayed feedback resulted in a significantly slower overall time and changed kinematic parameters, suggesting a tight link between auditory perception and action.

An interesting study that combined online and offline perception of own movements, was conducted by Keller, Knoblich and Repp [40]. In this study nine skilled musical ensemble players had to synchronize their actual movements with own past actions. Additionally, they were asked to recognize their own past performances. In the first recording session participants had to play one part of four different piano duets. After three to four months the players were asked to play the converse part of those music pieces that were formerly played by themselves or by another person. Players had no information whether they synchronized with own or other individuals movements. However, later on they were asked to identify their own former recordings. Interestingly the results of the present study provide evidence that the musical ensemble players showed better performances 
when they were asked synchronize with their own earlier performances. Additionally, they were able to identify their own past recordings. Furthermore, those piano players that showed high performance accuracy to synchronize with own past recordings were those participants that were better able to recognize own past performances. The results provide evidence that the simulation of a certain movement seems to be best for own past movements and therefore the piano players showed the best performances when they played with themselves.

Taken together, online and offline perception of own and other individuals' movements occurs in a variety of auditory contexts. Tempo and timing can be useful cues to identify actions (e.g. direction, gender) or an action's agent. However, these cues do not seem to be the only ones and the different results of the presented studies suggest that judgments are largely dependent of the type of manipulation and the nature of the task [for a review in the music domain coming to similar conclusions, see 41].

\section{THE ROLE OF AUDITORY CUES FOR ACTION CONTROL: THE FEEDBACK TYPE OF ACOUSTIC INFORMATION (INTERNAL NATURAL VS EXTER- NAL ARTIFICIAL)}

Auditory information can not only be used for the perception of different movements but also to control actions. As the previous sections have shown, many different sound types have been used to demonstrate the processing of acoustic action perception. If acoustic information is used as a type of feedback for optimizing performance, individuals usually have different options. Either they make use of their internal feedback system, namely the different senses, or they receive external feedback from others, such as coaches or spectators. With regard to acoustic feedback, internal feedback is only possible by perceiving the movement sounds that naturally occur from action execution. For instance, Agostini, Righi, Galmonte, and Bruno [42] showed that hammer throwers optimized their performance via training with auditory feedback. Taking the best personal throw, the sounds were generated by the movement of the hammer flying through the air and then played back to the participants while training. The athletes all improved and standardized their performance. As presented in the previous sections, recent research confirmed that naturally occurring movement sounds in form of auditory reafferences contain useful information in many respects [e.g. 27, 28].

With regard to external feedback that is directly related to movement, a method called sonification has been shown to be effective. Sonification is described as synthetically generated acoustic information, based on motion data $[43,44]$. By displaying kinematic parameters acoustically, numerical data is made audible. Usually acceleration measures of material (e.g. boat during rowing) or body parts (e.g. arms during breaststroke) are recorded, mapped to specific tones on the musical tone scale and then related to pitch. When recording movement sounds, biomechanical parameters such as angles cannot be perceived from sounds. Therefore, sonification enables auditory access to otherwise mute phases of the movement. Studies have shown that individuals are able to correctly perceive and identify movement patterns through sonification $[45,46]$ as well as using sonification to support the learning processes [46, 47]. For instance, in gymnastics, the circles performance on a pommel horse increased significantly, when this feedback system was used during training, which provided trajectory information in form of an auditory signal [47]. Since this review focuses on natural movement sounds, we recommend a systematic review by Dubus and Bresin [48] for further and more detailed information on artificial movement sounds generated through sonification.

In an attempt to compare the effect of natural versus artificial sounds on action perception and motor control, Thomas and Shiffrar [49] showed visual displays of human walking to participants. Detection sensitivity increased when visual displays were paired with veridical auditory cues (footfalls), but not with simple tones. The authors nicely summarized the results in their very appropriate title of the article: "I can see you better if I can hear you coming". The results were confirmed by a second experiment [37], leading to the conclusion that the use of auditory cues might only apply to meaningful sounds or even natural movement sounds. Similar results were revealed in a study in which participants were exposed to either ecological, artificial or no sounds of breathing, while measuring participants' breath duration variability [50]. The results showed that ecological sounds influenced the participants' breathing more than artificial sounds. This suggests that the ecological sounds seem to have been capturing the timing better, resulting in a more efficient use for action perception and motor control.

\section{NEURAL BASES OF AUDITORY ACTION PERCEP- TION}

Neurophysiological studies have been conducted to understand what happens in the brain during the auditory perception of action. Most of the studies in this domain have been conducted with musicians (especially pianists and string musicians), since they create sound patterns by their hand movements and therefore show tight couplings between sound and movement. The majority of these studies suggest that there is some form of "action-listening" or "hearingdoing" mechanism in individuals [51-54]. For instance, nonmusicians were trained to play a piece of music by ear (without notes) [55]. The behavioral data in this study revealed that participants increased their pitch recognition from $24 \%$ to $77 \%$ as a byproduct of learning to play by ear. Using fMRI, brain activity was monitored while the same participants listened to the newly acquired piece. Although participants did not perform any actions while listening, activation was found bilaterally in the frontoparietal motorrelated network. This coincided with the neural circuits related to action observations. If the notes were presented in a different order or equally familiar, but motorically unknown, this network was much less activated or not at all. Similar results were reported in an fMRI study involving tapping actions accompanying musical rhythms [56].

In studies using more complex whole-body movements, researchers investigated the neural substrate of action perception while listening to footsteps [17]. Besides activation in the auditory cortex, an auditory attentional network was shown sharing frontal and parietal areas previously found in visual attention paradigms. In addition, there was activation in a posterior superior temporal sulcus (STSp) region, overlapping the temporal human motion area as found with vis- 
ual input. The authors proposed that an area of the STSp region might be 'supramodal', independent of the sensory modality input. Similarly, another study also found that the STS responds to visual, auditory and tactile stimulation [57]. These and similar findings were summarized and reviewed in a chapter on multisensory-based approach by Làdavas [58]. The author concluded that similar and/or neighboring brain regions as in unimodal perception process the integration of different sensory modality inputs. One function of such multisensory-integrative networks, in which the same brain areas are activated during action perception and different modalities, might be the facilitation of movement patterns, as the behavioral studies, presented in the previous section, have demonstrated.

\section{CONCLUSION}

Understanding how we perceive motion across the different sensory domains is crucial given the multisensory environment within which we are immersed. The idea behind this review was to provide an overview about research conducted in the field of acoustic motor control and action perception. The review focused on three main dimensions regarding the use of auditory information, namely authorship (own/other), timing (offline/online) and types of feedback (internal/external). Taken together, the review identified the following patterns and trends in the literature: First, a large body of research has been realized to examine how auditory cues are perceived with regard to actions where the primary aim is to produce a certain auditory cue (e.g. hand clapping, piano playing). Up to now there are only a few attempts to examine how natural sounds occurring as a byproduct of a certain movement (e.g. walking) are used for actor identification and movement control. Besides walking, even less research has been committed to complex whole-body movements. Second, research aims are similar to those in visual perception research, where a reduction of a point-light figure to only a few cues is used to examine which information is used to identify an actor or the effect of a certain action [e.g. 59]: researchers investigate which component of the auditory information is used for actor identification [e.g. 28]. Third, research in the neuroscientific field examines what happens in the brain during the auditory perception of action. Not much is done regarding the question whether acoustic information that derives from an own movement leads to a different activation within certain brain areas compared to the perception of an auditory cue that belongs to another person's movement [but see 60].

There are several research gaps that need to be addressed in future research: First, up to now it seems not entirely clear which components or even which combination of components of acoustic information are used for action perception and control [28]. Further research should not only try to focus on the question which components of the auditory cue are used to identify a certain action or actor or which component is used to control the movement. Rather, given that there is a strong interaction of perception and action and both are coded on the same medium, research should much more focus on the question whether the components or mechanisms used for action perception are the same as those used for motor control. For instance, from a neuroscientific point of view, an interesting suggestion has been made to unravel these mechanisms for the visual domain [61]. Distinct neural pathways have been suggested for visual perception, differing between vision-for-perception and vision-for-action. Specifically, the "ventral pathway" in the temporal lobe is responsible for object recognition and identification, representing a viewer-independent mechanism for slower, but long-lasting object processing. The "dorsal pathway" in the parietal lobe applies to the orientation and location of an object, enabling dynamic visual information processing for fast and continuous motor control.

Furthermore, from a practical point of view it would be interesting to know which single component or combination of components of the auditory information is most useful to train and optimize certain movements in sports or rehabilitation. Second, similar to studies already conducted regarding the perception of own past movements based on visual cues [62], future research should examine what happens in the brain when listening to one's own past movements compared to other individuals' movements. Such research could provide evidence whether the postulated motor and perceptual resonance [3] can be observed on a cortical level.

To sum up, understanding online and offline perception of own and other individuals' movements with our different senses is crucial given the multisensory environment within which we interact on a daily basis. We have shown that research considers human perceptions of the world and perception-action integration processes as inherently cross-modal. Especially the so far underresearched area of auditory action perception and control offers interesting results that can be well linked to visual action perception and control. Our hurdler preparing for a competition might therefore consider training beside an Olympic gold medal winner in hurdling, to receive optimized visual as well as auditory information, to which he could attempt to adapt his own motor program.

\section{CONFLICT OF INTEREST}

The authors confirm that this article content has no conflict of interest.

\section{ACKNOWLEDGEMENTS}

Declared none.

\section{REFERENCES}

[1] Blake R, Shiffrar M. Perception of human motion. Ann Rev Psychol 2007; 58: 47-73.

[2] Schubotz RI. Prediction of external events with our motor system: towards a new framework. Trends Cogn Sci 2007; 11(5): 211-8.

[3] Schütz-Bosbach S, Prinz W. Perceptual resonance: action-induced modulation of perception. Trends Cogn Sci 2007; 11(8): 349-55.

[4] Hommel B, Müsseler J, Aschersleben G, et al. The Theory of Event Coding (TEC): A framework for perception and action planning. Behav Brain Sci 2001; 24: 849-937.

[5] Von Holst E, Mittelstaedt H. Das reafferenzprinzip. Naturwissenschaften 1950; 37: 464-76.

[6] Blakemore SJ, Frith CD, Wolpert DM. Spatiotemporal prediction modulates the perception of self-produced stimuli. J Cogn Neurosci 1999; 11: 551-9.

[7] Anderson JR, Lebiere C. The atomic components of thought. NJ: Lawrence Erlbaum Associates 1998.

[8] Prinz W. Perception and action planning. Eur J Cogn Psychol 1997; 9(2): 129-54. 
[9] Kilner JM, Friston KJ, Frith CD. The mirror-neuron system: a Bayesian perspective. Neuroreport 2007; 18(6): 619-23.

[10] Rizzolatti G, Craighero L. The mirror-neuron system. Ann Rev Neurosci 2004; 27: 169-92.

[11] Jeannerod M. Neural simulation of action: a unifying mechanism for motor cognition. NeuroImage 2001; 14: 103-9.

[12] Rizzolatti G, Fogassie L, Gallese V. Neurophysiological mechanisms underlying the understanding and imitation of action. Nat Rev Neurosci 2001; 2: 661-70.

[13] Dietrich A. Imaging the imagination: The trouble with motor imagery. Methods 2008; 45: 319-24.

[14] MacPherson AC, Collins D, Obhi SS. The importance of temporal structure and rhythm for the optimum performance of motor skills: A new focus for practitioners of sport psychology. J Appl Sport Psychol 2009; 21: 48-61.

[15] Ekimov A, Sabatier JM. Vibration and sound signatures of human footsteps in buildings. J Acoust Soc Am 2006; 120: 762.

[16] Saarela MV, Hari R. Listening to humans walking together activates the social brain circuitry. Soc Neurosci 2008; 3: 401-9.

[17] Bidet-Caulet A, Voisin J, Bertrand O, et al. Listening to a walking human activates the temporal biological motion area. Neuroimage 2005; 28: 132-9.

[18] Pastore RE, Flint JD, Gaston JR, et al. Auditory event perception: The source-perception loop for posture in human gait. Percept Psychophys 2008; 70(1): 13-29.

[19] Li X, Logan RJ, Pastore RE. Perception of acoustic source characteristics: Walking sounds. J Acous Soc1991; 90(6): 3036-49.

[20] Cottrell D, Campbell MEJ. Auditory perception of a human walker. Perception 2014; 43(11): 1225-38.

[21] Grèzes J, Frith $\mathrm{CD}$, Passingham RE. Inferring false beliefs from the actions of oneself and others: an fMRI study. NeuroImage 2004; 21: 744-50.

[22] Loula F, Prasad S, Harper K, et al. Recognizing people from their movement. J Exp Psychol-Hum Percept Perform 2005; 31(1): 21020.

[23] Repp BH. The sound of two hands clapping: An exploratory study. J Acoust Soc Am 1987; 81: 1100-9.

[24] Flach R, Knoblich G, Prinz W. Recognizing one's own clapping: The role of temporal cues. Psychol Res 2004; 69: 147-56.

[25] Repp BH, Knoblich G. Perceiving action identity: How pianists recognize their own performances. Psychol Sci 2004; 15: 604-9.

[26] Murgia M, Hohmann T, Galmonte A, et al. Recognising one's own motor actions through sound: The role of temporal factors. Perception 2012; 41: 976-87.

[27] Kennel C, Hohmann T, Raab M. Action perception via auditory information: Agent identification and discrimination with complex movement sounds. J Cogn Psychol 2014; 26(2): 157-65.

[28] Kennel C, Pizzera A, Hohmann T, et al. The perception of natural and modulated movement sounds. Perception 2014; 43: 796-804.

[29] Klapp ST, Jagacinski RJ. Gestalt principles in the control of motor action. Psychol Bull 2011; 137: 443-62.

[30] Righi G, Galmonte A, Agostini T. Rhythm, a Gestalt of human movement? Gestalt Theory 2006; 28: 283-91

[31] Casile A, Giese MA. Nonvisual motor training influences biological motion perception. Curr Biol 2006; 16: 69-74.

[32] Sato A, Yasuda A. Illusion of sense of self-agency: discrepancy between the predicted and actual sensory consequences of actions modulates the sense of self-agency, but not the sense of selfownership. Cognition 2005; 94(3): 241-55.

[33] Menzer F, Brooks A, Halje P, et al. Feeling in control of your footsteps: Conscious gait monitoring and the auditory consequences of footsteps. Cogn Neurosci 2010; 1(3): 184-92.

[34] Pfordresher PQ. Coordination of perception and action in music performance. Adv Cogn Psychol 2006; 2(2-3): 183-98.

[35] Arrighi R, Marini F, Burr D. Meaningful auditory information enhances perception of visual biological motion. J Vis 2009; 9(4): 25.1-7.
[36] Sevdalis V, Keller PE. Cues for recognition in point-light displays of action performed in synchrony with music. Conscious Cogn 2010; 19: 617-26.

[37] Thomas JP, Shiffrar M. Meaningful sounds enhance visual sensitivity to human gait regardless of synchrony. J Vis $2013 ; 13(14)$ : 113.

[38] Cesari P, Camponogara I, Papetti S, et al. Might as well jump: sound affects muscle activation in skateboarding. PLoS One 2014; 9(3): 1-9.

[39] Kennel C, Streese L, Pizzera A, et al. Auditory reafferences: the influence of real-time feedback on movement control. Front Psychol 2015; 6(69): 1-6.

[40] Keller P, Knoblich G, Repp B. Pianists duet better when they play with themselves: On the possible role of action simulation in synchronization. Conscious Cogn 2007; 16(1): 102-11.

[41] Sevdalis V, Keller PE. Know thy sound: Perceiving self and others in musical contexts. Acta Psychologica 2014; 152: 67-74.

[42] Agostini T, Righi G, Galmonte A, et al. The relevance of auditory information in optimizing hammer throwers performance. In: Pascolo PB, Ed. Biomechanics and sports. Vienna, Austria: Springer 2004; pp. 67-74.

[43] Kramer G. Auditory display: Sonification, audification, and auditory interfaces. Santa Fe Institute Studies in the Sciences of Complexity, Proceedings, vol. XVIII. US: Addison Wesley Publishing Company, Reading 1994.

[44] Schaffert N, Mattes K, Effenberg AO. A sound design for acoustic feedback in elite sports. In: Ystad S, Aramaki M, KronlandMartinet R, Jensen K, Eds. Auditory Display. $6^{\text {th }}$ International Symposium, CMMR/ICAD 2009. Berlin: Springer-Verlag 2010.

[45] Effenberg AO, Mechling H. Movement-sonification: A new approach in motor control and learning. J Sport Exerc Psychol 2005; 27: 58-68.

[46] Schaffert N, Mattes K, Effenberg AO. An investigation of online acoustic information for elite rowers in on-water training conditions. J Hum Sport Exerc 2011; 6: 392-405.

[47] Baudry L, Leroy D, Thouvarecq R, Chollet D. Auditory concurrent feedback benefits on the circle performed in gymnastics. J Sport Sci 2006; 24: 149-56.

[48] Dubus G, Bresin R. A systematic review of mapping strategies for the sonification of physical quantities. PLoS One 2013; 8(12): 128 .

[49] Thomas JP, Shiffrar M. I can see you better if I can hear you coming: Action-consistent sounds facilitate the visual detection of human gait. J Vis 2010; 10: 1-11.

[50] Murgia M, Santoro I, Tamburini G, et al. Ecological sounds affect breath duration more than artificial sounds. Psychol Res 2015; [Epub ahead of Print].

[51] Bangert M, Peschel T, Schlaug G, et al. Shared networks for auditory and motor processing in professional pianists: evidence from fMRI conjunction. Neuroimage 2006; 30: 917-26.

[52] Baumann S, Koeneke S, Schmidt CF, et al. A network for audiomotor coordination in skilled pianists and non-musicians. Brain Res 2007; 1161: 65-78.

[53] Haslinger B, Erhard P, Altenmüller E, et al. Transmodal sensorimotor networks during action observation in professional pianists. J Cogn Neurosci 2005; 17: 282-93.

[54] Lotze M, Scheler G, Tan HRM, et al. The musician's brain: functional imaging of amateurs and professionals during performance and imagery. Neuroimage 2003; 20: 1817-29.

[55] Lahav A, Saltzman E, Schlaug G. Action representation of sound. J Neurosci 2007; 27(2): 308-14

[56] Chen JL, Penhune VB, Zatorre RJ. Listening to musical rhythms recruits motor regions of the brain. Cereb Cortex 2008; 18: 284454 .

[57] Zatorre RJ, Chen JL, Penhune VB. When the brain plays music: auditory-motor interactions in music perception and production. Nat Rev Neurosci 2007; 8(7): 547-58. 
[58] Làdavas E. Multisensory-based approach to the recovery of unisensory deficit. Ann N Y Acad Sci 2008; 1124: 98-110.

[59] Munzert J, Hohmann T, Hossner EJ. Discriminating throwing distances from point-light displays with masked ball flight. Eur J Cogn Psychol 2010; 22: 247-64.

[60] Justen C, Herbert C, Werner K, et al. Self vs. Other: Neural correlates underlying agent identification based on unimodal auditory in- formation as revealed by electrotomography (sLORETA). Neuroscience 2014; 259: 25-34.

[61] Goodale MA, Króliczak G, Westwood DA. Dual routes to action contributions of the dorsal and ventral streams to adaptive behavior. Prog Brain Res 2005; 149: 269-83.

[62] Bischoff M, Zentgraf K, Pilgramm S, et al. Motor familiarity: brain activation when watching kinematic displays of one's own movements. Neuropsychologia 2012; 50(8): 2085-92.

Received: September 22,2015

Revised: November 03,2015

Accepted: November 03,2015

(C) Pizzera and Hohmann; Licensee Bentham Open.

This is an open access articles licensed under the terms of the Creative Commons Attribution-Non-Commercial 4.0 International Public License (CC BY-NC 4.0) (https://creativecommons.org/licenses/by-nc/4.0/legalcode), which permits unrestricted, non-commercial use, distribution and reproduction in any medium, provided that the work is properly cited. 